Original paper

\title{
Metabolic associated fatty liver disease and adverse maternal and fetal outcomes: a systematic review and meta-analysis
}

\author{
Adinda Ayu Dyah, Rahadina Rahadina \\ Faculty of Medicine, Universitas Airlangga, Surabaya, Indonesia
}

\begin{abstract}
Aim of the study: Metabolic-associated fatty liver disease (MAFLD) is now the most prevalent chronic liver disease in the world. Its prevalence in women of reproductive age is approximately $10 \%$. Due to its high prevalence in this specific population, it is important to investigate adverse maternal and fetal outcomes caused by MAFLD during pregnancy. We aimed to summarize the association between MAFLD and adverse maternal and fetal outcomes.
\end{abstract}

Material and methods: A search was conducted on PubMed and ProQuest from inception to April 1, 2021, for studies assessing the association between MAFLD and adverse maternal and fetal outcomes. The quality of included studies was assessed using the Newcastle-Ottawa scale (NOS). We analyzed the pooled odds ratios (ORs) with 95\% confidence intervals ( $\mathrm{Cl}$ ) using a fixed and random-effects model. Heterogeneity was assessed using $P$.

Results: Six studies comprising 20,535,994 (5,964 MAFLD) pregnant women were included. The quality of studies ranged from 6 to 8 stars. MAFLD was significantly associated with increased risk of dysglycemia $(O R=3.65$, $95 \% \mathrm{Cl}=2.47-5.39)$, pregnancy-associated hypertension $(\mathrm{OR}=3.27,95 \% \mathrm{Cl}=2.75-3.88)$, cesarean section $(\mathrm{OR}=2.78,95 \% \mathrm{Cl}=1.60-4.83)$, and preterm birth $(\mathrm{OR}=1.70,95 \% \mathrm{Cl}=1.37-2.10)$ but not large for gestational age $(\mathrm{OR}=1.69,95 \% \mathrm{Cl}=0.64-4.45)$.

Conclusions: The presence of MAFLD is associated with adverse maternal and fetal outcomes.

Key words: metabolic-associated fatty liver disease, pregnancy, systematic review, meta-analysis.

Address for correspondence:

Dr. Adinda Ayu Dyah, Faculty of Medicine, Universitas Airlangga, Surabaya, Indonesia, e-mail: adinda.ayu-13@fk.unair.ac.id

\section{Introduction}

Metabolic associated fatty liver disease (MAFLD) is a new nomenclature for nonalcoholic fatty liver disease (NAFLD), with hepatic steatosis and metabolic dysfunction as its hallmark [1]. It comprises a wide clinical spectrum, from simple steatosis to a more progressive form, steatohepatitis with different degree of fibrosis. Central obesity, diabetes mellitus, hypertension (HTN), and hyperlipidemia are all linked to this disease [2]. MAFLD develops as a result of various deposits of activated proinflammatory cytokines in the liver, such as tumor necrosis factor $\alpha$ and interleukin 6 , which is promoted by excess free fatty acids, hepatic insulin resistance, and adipose tissue in the periphery [3].
MAFLD is now the most common liver disease in the world, with a prevalence of $20-30 \%$, and is becoming the major cause of liver transplantation in Western populations [1]. Its prevalence is increasing, but variation exists across studies due to differences in frequency of obesity in the study population, age, and diagnostic criteria [4]. In women at productive age, prevalence is estimated at $10 \%$, and it is highly associated with the most prevalent disorder at their age, polycystic ovarian syndrome $[5,6]$.

With such high prevalence, it is important to investigate the magnitude of adverse maternal and fetal outcomes that will be inflicted by MAFLD occurring during pregnancy. Our aim was to summarize the association between MAFLD and adverse maternal and fetal outcomes. 
Table 1. Search strategy

\begin{tabular}{|c|c|c|}
\hline Search & Query & Results \\
\hline \multicolumn{3}{|l|}{ PubMed } \\
\hline$\# 1$ & ((“Non-alcoholic Fatty Liver Disease"[Mesh]) OR ("nafld"[All Fields])) OR ("non alcoholic fatty liver disease"[All Fields]) & 23,867 \\
\hline \#2 & (“Pregnancy"[Mesh]) OR ("pregnancy"[All Fields]) & $1,007,060$ \\
\hline \#3 & \#1 AND \#2 & 252 \\
\hline \multicolumn{3}{|l|}{ ProQuest } \\
\hline S1 & (SU.exact(“'LIVER DISEASES”) AND SU.exact(“FATTY LIVER”)) & 5,503 \\
\hline S2 & $\begin{array}{c}\text { (MAINSUBJECT.EXACT(“Pregnancy”) } \\
\text { OR MESH.EXACT(“Pregnancy”)) }\end{array}$ & 121,409 \\
\hline S3 & 1 AND 2 & 44 \\
\hline
\end{tabular}

ACOG - American College of Obstetricians and Gynecologists, BMI - body mass index, CS - caesarean section, DBP - diastolic blood pressure, FGR - fetal growth restriction, GDM - gestational diabetes mellitus, HOMA-IR - homeostatic model assessment for insulin, HTN - hypertension, ICD - International Classification of Diseases, IFG - impaired fasting glucose, IGT - impaired glucose tolerance, LGA - large for gestational age, MAFLD - metabolic associated fatty liver disease, NA - not applicable, OGTT - oral glucose tolerance test, PE - pre-eclampsia, PTB - preterm birth, SBP - systolic blood pressure, SeP - selenoprotein, SGA - small for gestational age, T2DM - type 2 diabetes mellitus, TG - triglyceride, US - ultrasonography, WC - waist circumference

\section{Material and methods}

\section{Study selection}

Our meta-analysis adhered to Preferred Reporting Items for Systematic Reviews and Meta-Analyses (PRISMA) guidelines. Two independent investigators conducted a literature search on PubMed and ProQuest from inception to April 1, 2021, restricting the search to studies involving humans and articles published in English. We used medical subject headings and free-text terms for the keywords, as shown in Table 1.

\section{Eligibility criteria}

We included studies if they met the following criteria: 1) the study was a cohort or case-control study, 2) the study estimated the association between the presence of MAFLD and the occurrence of adverse maternal (pregnancy-associated dysglycemia, pregnancy-associated HTN, and cesarean section [CS]) and fetal outcomes (preterm birth [PTB] and large for gestational age [LGA]), 3) the study reported odds ratios (ORs) with their respective $95 \%$ confidence intervals (CIs) or presented raw data. When the study carried out analyses with adjustment for confounding factors, we chose the adjusted over unadjusted OR. Literature was excluded in the following cases: 1) short communications, letters to the editor, conference abstracts, reviews, case reports, or cross-sectional studies, 2) desirable data could not be retrieved, 3) full-text articles were not available, 4) non-English articles.

\section{Study quality}

The risk of bias of included studies (both cohort and case-control studies) was separately assessed by two investigators using the Newcastle-Ottawa scale (NOS). Studies with a NOS score $<7$ were considered to have a high risk of bias, whereas those with a score $\geq 7$ were considered to have a low risk of bias.

\section{Data extraction}

The same two investigators independently extracted the following data from each study using a prespecified form which consists of author, year, study design, country, sample size, MAFLD diagnosis criteria, outcome, and adjustment used.

\section{Statistical analysis}

We estimated the impact of presence of MAFLD on maternal and fetal outcomes through pooled OR with their corresponding 95\% CI. Data were pooled based on the fixed-effects or random-effects assumption. $P$-value $<0.05$ was considered to be significant. Higgins' $I^{2}$ statistic was used to assess heterogeneity. If the value of the $I^{2}$ statistic was $<50 \%$, a fixed-effect model could be applied. Otherwise, a random-effect model could be used. We planned to assess publication bias through a funnel plot if included studies reached the minimum number of 10 . An asymmetric plot could suggest publication bias. All statistical analyses were performed using Review Manager version 5.4 (The Cochrane Collaboration).

\section{Results}

Of the 296 potential records that were initially identified by the search strategy, 236 were discarded after removing duplicates and by eligibility screening of titles and abstracts. After further full-text examina- 
tions for 60 articles, six studies were considered eligible for qualitative and quantitative synthesis (Fig. 1).

\section{Study characteristics}

The baseline characteristics of the six studies are summarized in Table 2. The studies involved 20,535,994 (5,964 MAFLD) pregnant women, ranging from 476 to $18,574,225$ patients in each study, originating from 4 different countries. Of 6 included studies, three studies took place in South Korea (which were derived from one big study), one study was from Sweden, and the remaining studies were from Canada and the United States. Studies were published between 2015 and 2020. Of six studies, four studies diagnosed MAFLD through US at 10-14 weeks of gestation while two studies used diagnostic codes from the national patient registry. Four studies reported the risk of pregnancy-associated dysglycemia. Three studies reported the outcome of gestational diabetes mellitus and one study reported a composite outcome of gestational diabetes mellitus (GDM) with other abnormal findings in the oral glucose tolerance test (OGTT), which were impaired fasting glucose (IFG) and impaired glucose tolerance (IGT) [7]. Three studies reported the pregnancy-associated HTN outcome. Outcomes of CS and PTB were both reported by Hagström et al. [8] and Sarkar et al. [9]. Another fetal outcome, LGA, was reported by two studies.

\section{Quality of included studies}

As seen in Table 3, the study quality ranged from 6 to 8 . Five studies were considered at low risk of bias, while one was deemed to have a high risk of bias.

\section{Publication bias}

We could not conduct publication bias assessment through funnel plot as the number of included studies was 6.

\section{MAFLD and maternal outcomes}

Studies investigating the association between MAFLD and pregnancy-associated dysglycemia are scarce. Pooled results on presence of MAFLD and the risk of pregnancy-associated dysglycemia are shown in Figure 2. Presence of MAFLD was significantly associated with subsequent dysglycemia (pooled $\mathrm{OR}=3.65$, $95 \% \mathrm{CI}=2.47-5.39, p<0.01)$. A moderate amount of heterogeneity was observed $\left(I^{2}=51 \%, p=0.10\right)$.

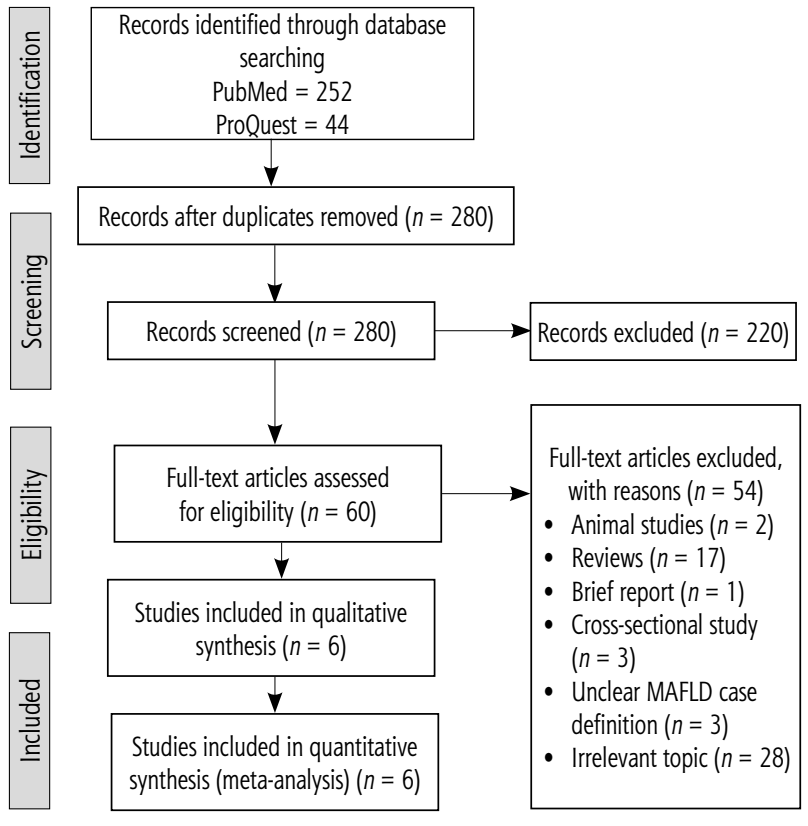

Fig. 1. PRISMA flow diagram

MAFLD was also significantly associated with the odds of developing pregnancy-associated HTN and CS. Pooled results on presence of MAFLD and the risk of developing pregnancy-associated HTN and CS are shown in Figure 3 and 4, respectively (pooled $\mathrm{OR}=3.27,95 \% \mathrm{CI}=2.75-3.88, p<0.01$ and pooled $\mathrm{OR}=2.78,95 \% \mathrm{CI}=1.60-4.83, p<0.01)$. While no heterogeneity was observed in studies reporting pregnancy-associated HTN, a considerable amount of heterogeneity was present in CS data $\left(I^{2}=88 \%, p<0.01\right)$.

\section{MAFLD and fetal outcomes}

Studies evaluating MAFLD and fetal outcomes are much more limited than those evaluating maternal outcomes. Two studies investigated the outcome of PTB. A significant association was found between maternal MAFLD and PTB, shown in Figure 5, with no heterogeneity $(\mathrm{OR}=1.70,95 \% \mathrm{CI}=1.37-2.10$, $\left.I^{2}=47 \%\right)$.

Another fetal outcome reported was LGA. Our pooled results found that MAFLD was not significantly associated with an increase odds of LGA, as shown in Figure 6 (pooled OR $=1.69,95 \% \mathrm{CI}=0.64-4.45$, $\left.I^{2}=73 \%\right)$.

\section{Discussion}

The current research summarized the association between maternal MAFLD and adverse maternal and fetal outcomes from 6 observational studies. From our pooled results, MAFLD was significantly correlated 
Table 2. Study characteristics

\begin{tabular}{|c|c|c|c|}
\hline $\begin{array}{l}\text { Author, year, } \\
\text { study design, } \\
\text { country, sample } \\
\text { size }\end{array}$ & MAFLD diagnostic criteria & Outcome & Adjustment used \\
\hline $\begin{array}{l}\text { De Souza, } 2016 \\
\text { [34], cohort, } \\
\text { Canada, } 476\end{array}$ & $\begin{array}{l}\text { MAFLD was diagnosed using US at 11-14 } \\
\text { weeks of gestation. Semiquantitative scoring } \\
\text { was used by two independent sonographers, } \\
\text { based on the finding of } \\
\text { (i) diffusely increased echogenic ("bright") } \\
\text { liver greater than the right kidney } \\
\text { ("hepatorenal contrast") and/or (ii) impaired } \\
\text { visualization (blurring) of the portal and } \\
\text { hepatic veins. Each pregnant woman was } \\
\text { scored as "0", "1", or "2" according to the } \\
\text { number of findings. MAFLD was then } \\
\text { diagnosed if } \geqslant 1 \text { finding was present }\end{array}$ & $\begin{array}{c}\text { Composite outcome of IFG, IGT, or GDM at } 24-28 \text { weeks, } \\
\text { by a fasting } 75 \mathrm{~g} \text { OGTT. IFG was defined as fasting glucose } \\
\geqslant 5.3 \mathrm{mmol} / \mathrm{l} \text {. IGT was defined as glucose value at } 1 \text { hour } \\
\geqslant 10.6 \mathrm{mmol} / \mathrm{l} \text {. GDM was defined as fasting glucose } \\
\geqslant 5.3 \mathrm{mmol} / \mathrm{l}, \text { glucose at } 1 \text { hour } \geqslant 10.6 \mathrm{mmol} / \mathrm{l}, \\
\text { and } / \text { or at } 2 \text { hour } \geqslant 8.9 \mathrm{mmol} / \mathrm{l}\end{array}$ & $\begin{array}{l}\text { Age, ethnicity, first-degree } \\
\text { relative with T2DM, BMI }\end{array}$ \\
\hline $\begin{array}{c}\text { Hagström, } \\
2015[9], \text { case } \\
\text { control, Sweden, } \\
1,960,416\end{array}$ & $\begin{array}{l}\text { Diagnostic code from national patient } \\
\text { registry (K76.0 in ICD-10 and 571W } \\
\text { in ICD-9) }\end{array}$ & $\begin{array}{l}\text { GDM (E10 and E11 in ICD-10 and 250A-X in ICD-9, } \\
\text { prior to first antenatal visit), PE, CS, and PTB } \\
\text { (gestational age } \leqslant 36 \text { at delivery) }\end{array}$ & $\begin{array}{l}\text { NA } \\
\text { Data were only available } \\
\text { as crude OR }\end{array}$ \\
\hline $\begin{array}{l}\text { Jung, } 2020[33] \\
\text { cohort, } \\
\text { South Korea, } \\
877\end{array}$ & $\begin{array}{l}\text { US at 10-14 weeks of gestation. MAFLD } \\
\text { was defined as the presence of a bright } \\
\text { echogenic pattern within the liver }\end{array}$ & $\begin{array}{l}\text { Pregnancy-associated HTN, consisting of gestational HTN, } \\
\text { PE, or eclampsia after } 20 \text { weeks of gestation, was defined } \\
\text { when measurement exceeded } 140 \mathrm{mmHg} \text { or } 90 \mathrm{mmHg} \text { for } \\
\text { SBP and DBP, respectively. Gestational HTN was defined as } \\
\text { HTN for the first time during pregnancy without proteinuria } \\
\text { or end-organ damage, while PE was with end-organ damage }\end{array}$ & $\begin{array}{l}\text { WC, DBP at liver US, fasting } \\
\text { glucose, and SeP }\end{array}$ \\
\hline $\begin{array}{l}\text { Lee, } 2019[16] \\
\text { cohort, } \\
\text { South Korea, } \\
608\end{array}$ & $\begin{array}{l}\text { US at 10-14 weeks of gestation. MAFLD } \\
\text { was defined as the presence of a bright } \\
\text { echogenic pattern within the liver }\end{array}$ & $\begin{array}{l}\text { GDM was assessed using two-step approach as per ACOG } \\
\text { recommendation. GDM was diagnosed when there were } \\
\geqslant 2 \text { elevated glucose levels (i.e. fasting glucose } \\
\geqslant 5.3 \mathrm{mmol} / \mathrm{l} \text {, glucose at } 1 \text { hour } \geqslant 10.6 \mathrm{mmol} / \mathrm{l} \text {, at } 2 \text { hours } \\
\geqslant 8.6 \mathrm{mmol} / \mathrm{l} \text {, and at } 3 \text { hours } 7.8 \mathrm{mmol} / \mathrm{l} \text { ) }\end{array}$ & $\begin{array}{c}\text { Age, history of GDM, WC, SBP, } \\
\text { DBP, HOMA-IR, adiponectin, } \\
\text { SeP }\end{array}$ \\
\hline $\begin{array}{l}\text { Lee, } 2019[26] \\
\text { cohort, South } \\
\text { Korea, } 623\end{array}$ & $\begin{array}{l}\text { US at 10-14 weeks of gestation. MAFLD } \\
\text { was defined as the presence of a bright } \\
\text { echogenic pattern within the liver }\end{array}$ & $\begin{array}{l}\text { LGA was obtained from birth records data (birthweight } \\
\qquad>90^{\text {th }} \text { percentile for gestational age) }\end{array}$ & $\begin{array}{l}\text { Age, GDM, pre pregnancy } \\
\text { BMI, TG, HOMA-IR }\end{array}$ \\
\hline $\begin{array}{l}\text { Sarkar, } 2020 \\
\text { [10], case } \\
\text { control, } \\
\text { United States, } \\
18,574,225\end{array}$ & Diagnostic code from national database & $\begin{array}{l}\text { GDM, pregnancy-associated HTN, CS, PTB, LGA, } \\
\text { all were obtained from national database }\end{array}$ & $\begin{array}{l}\text { Pregnancy-associated HTN, PTB } \\
\text { and LGA were adjusted for age, } \\
\text { race, multiple gestation, pre- } \\
\text { existing diabetes, HTN, } \\
\text { and maternal obesity } \\
\text { The outcome of GDM and CS } \\
\text { was not adjusted }\end{array}$ \\
\hline
\end{tabular}

with development of pregnancy-associated dysglycemia, pregnancy-associated HTN, CS, and PTB. However, we did not find LGA to be significantly correlated with maternal MAFLD.

The underlying mechanism of association between maternal MAFLD and subsequent dysglycemia and GDM is not fully elucidated. Obesity may be the intermediary link between MAFLD and GDM. MAFLD, with its new nomenclature, is now recognized as a metabolic syndrome that is strongly associated with obesity, a known risk factor for GDM development [10]. The presence of hepatic steatosis also indicates insulin resistance (IR), a subclinical condition which may further influence glucose metabolism derangement in seemingly healthy women during pregnancy and post-partum period [11]. Apart from obesity and IR, other mechanisms are likely to take part. A prior finding was that fat accumulation and later development of MAFLD may be the product of proinflammatory cytokines that are transported to the liver from other intra-abdominal visceral tissues [12, 13]. A 2018 Korean study found that levels of adipokines and selenoprotein $(\mathrm{SeP})$ were significantly correlated with both severity of MAFLD in the first trimester and risk of 
Table 3. Quality of included studies

\begin{tabular}{lcccc}
\hline Study & Selection & Comparability & Outcome/exposure & NOS score \\
\hline De Souza, 2016 [34] & 3 & 2 & 3 & 8 \\
\hline Hagström, 2015 [9] & 2 & 1 & 3 & 6 \\
\hline Jung, 2020 [33] & 3 & 2 & 3 & 8 \\
\hline Lee, 2019 [16] & 3 & 2 & 3 & 8 \\
\hline Lee, 2019 [26] & 3 & 2 & 3 & 8 \\
\hline Sarkar, 2020 [10] & 2 & 2 & 3 & 7 \\
\hline
\end{tabular}

NOS - Newcastle-Ottawa scale

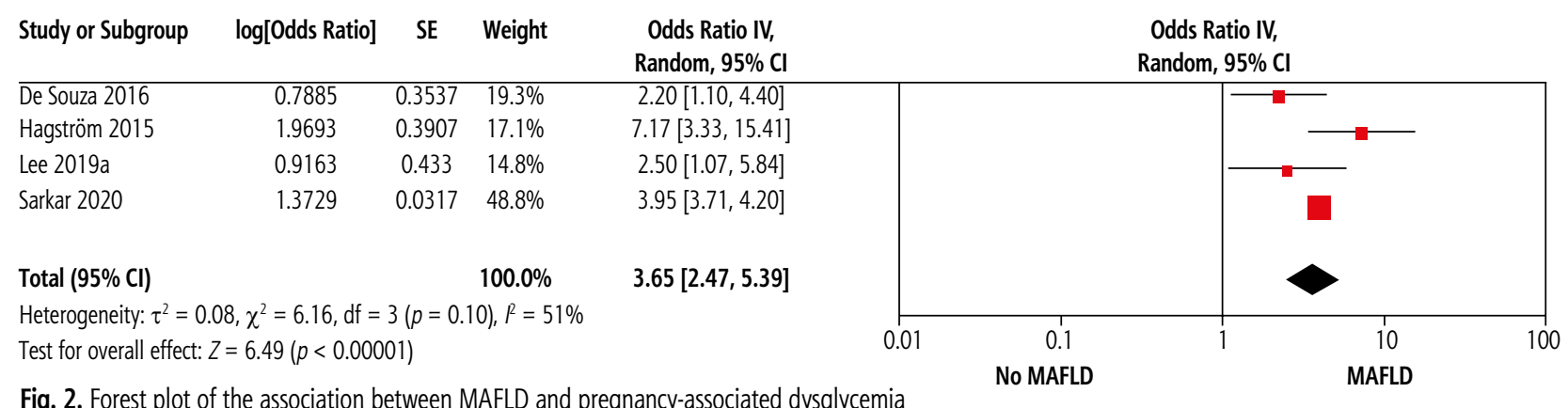

Fig. 2. Forest plot of the association between MAFLD and pregnancy-associated dysglycemia

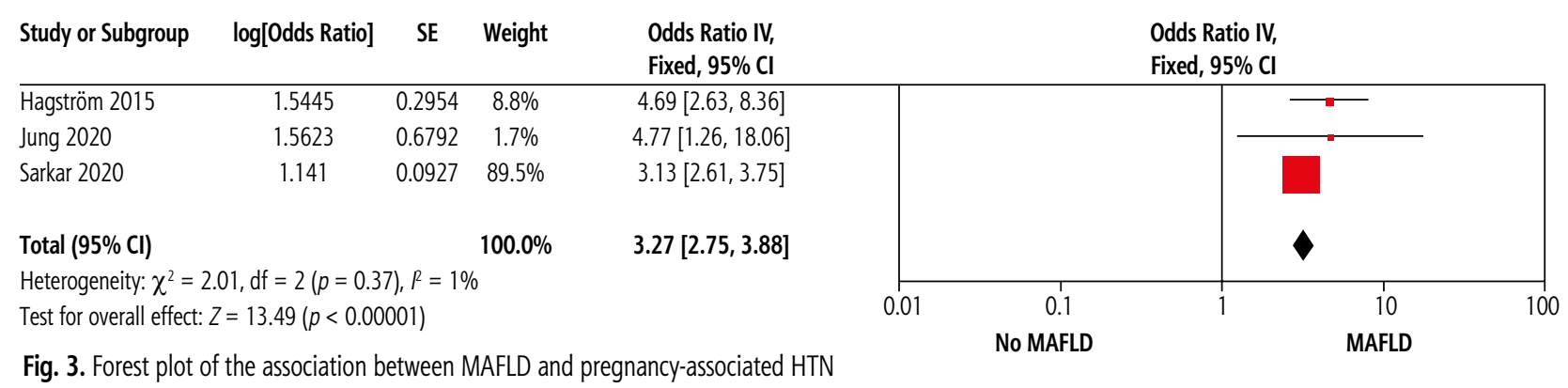

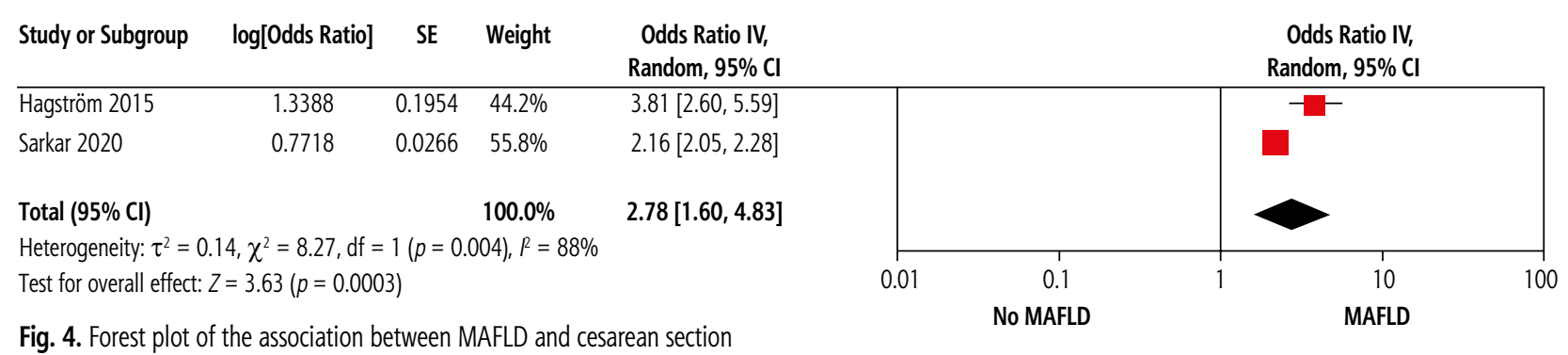

GDM development. The association even persisted after adjusting for potential confounders. This indicates that adipokine and SeP may be independent predictors of GDM development in MAFLD pregnant women and may have implications for its role in screening [14]. Although statistically significant, our study had a moderate amount of heterogeneity. This might be due to the fact that MAFLD and GDM diagnosis varied across studies. Two studies used US and two others used diagnostic codes from the national patient registry. Given the low prevalence of MAFLD in 1 registry study, the possibility of undetected cases is not to be missed. GDM diagnostic methods also varied across studies. Two studies used the national registry but did not state the methods used, 1 study used the 2-step method as per the American College of Obstetricians and Gynecologists (ACOG) recommendation, and 1 study used the $75 \mathrm{~g}$ OGTT. There has been much discussion on what is the superior approach in screening and diagnosing GDM. While the included studies were limited, a recent meta-analysis found no significant difference in GDM incidence between the two. However, 


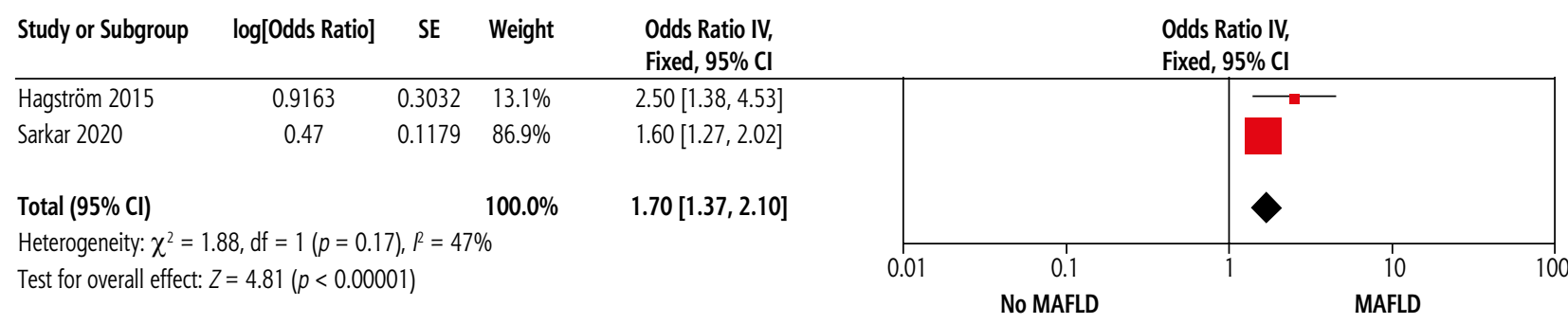

Fig. 5. Forest plot of the association between MAFLD and PTB

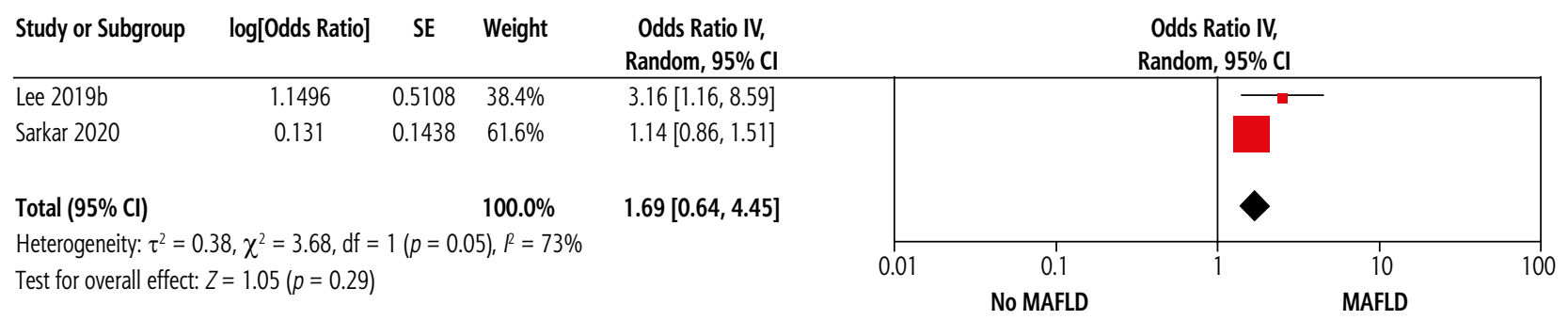

Fig. 6. Forest plot of the association between MAFLD and LGA

$75 \mathrm{~g}$ OGTT was found to be linked to better perinatal outcomes compared to the 2-step method [15].

Our study also showed a significant association between maternal MAFLD and pregnancy-associated HTN. A shifted composition of plasma monounsaturated fatty acids and a low level of linoleic acid are often present in MAFLD patients. One randomized controlled trial found that linoleic acid supplementation along with calcium possessed an ability to prevent the occurrence of preeclampsia. The association between maternal MAFLD and pregnancy-associated HTN could be explained by these findings [16]. Other proposed mechanisms are the sympathetic activation that is caused by IR and hyperinsulinemia, common among MAFLD patients, as well as vasodilation as a consequence of the dysfunction of insulin regulation. Hyperinsulinemia-induced sodium absorption may further enhance the development of $\operatorname{HTN}[17,18]$.

Increased odds of adverse fetal outcomes such as PTB were noted among MAFLD pregnant women. Maternal MAFLD is linked to maternal overweight and obesity, and thus confers a higher risk of preterm delivery [19].

Large for gestational age may occur as a result of fetal overgrowth due to a surge of maternal glucose and free fatty acid release, which exists in GDM [20]. Not only may LGA complicate a delivery by the increased risk of shoulder dystocia and birth trauma, but it also potentially deranges infant metabolism through all life stages, as manifested by an increased risk of childhood MAFLD and diabetes development at a later age [21, 22 ]. Study by Lee et al. found that the association persisted after adjusting for GDM status, which indicates that maternal MAFLD is an independent predictor of
LGA [20, 23]. However, our pooled result did not show any significant correlation between LGA and maternal MAFLD.

Because of the strong association that maternal MAFLD has with the risk of adverse maternal and fetal outcomes, an effort to detect MAFLD in pregnant women is needed. Choosing a safe tool with acceptable diagnostic accuracy for diagnosing MAFLD in pregnant women is a challenge. While liver biopsy is the gold standard for diagnosing MAFLD, its application in pregnant women with no apparent symptoms may not be justified. US has limited sensitivity and specificity for diagnosing MAFLD in obese patients, especially for a liver content below 12.5\% [24]. However, given its availability and safety, it may seem to be the modality of choice for now. A US-based method, transient liver elastography (TLE), has also been studied before. While TLE has been widely recognized for its use on nonpregnant populations, primarily because of its ability to detect milder fatty liver and quantify the degree of fibrosis as opposed to US, data on pregnant women are scant [25-29]. The use of other noninvasive scores, mainly composed from blood studies, has also emerged. The fatty liver index and the hepatic steatosis index are examples of noninvasive scores used in prior studies. Clearly, it needs further validation in pregnant women $[14,23,30]$.

\section{Limitation}

To the best of our knowledge, this is the first meta-analysis to quantify the association between presence of MAFLD and adverse maternal and fetal outcomes. However, our meta-analysis has some 
limitations. First, the number of studies was limited. Second, the methods of MAFLD diagnosis varied and could have contributed to the bias. Four studies used US, while two other studies used the national patient registry. Although substantial heterogeneity was found in a few variables, our research shows promising evidence and the urgent need to include MAFLD as a subgroup of patients who will benefit from a high-risk obstetric team's evaluation. Further longitudinal studies with diagnostic tools other than or a combination with US, carrying better diagnostic accuracy, are warranted to elucidate the association between maternal MAFLD and adverse maternal and fetal outcomes.

\section{Conclusions}

Presence of MAFLD is associated with adverse maternal and fetal outcomes.

\section{Disclosure}

The authors declare no conflict of interest.

\section{References}

1. Eslam M, Sanyal AJ, George J. MAFLD: a consensus-driven proposed nomenclature for metabolic associated fatty liver disease. Gastroenterology 2020; 158: 1999-2014.e1.

2. Armstrong MJ, Adams LA, Canbay A, Syn WK. Extrahepatic complications of nonalcoholic fatty liver disease. Hepatology 2014; 59: 1174-1197.

3. Peverill W, Powell LW, Skoien R. Evolving concepts in the pathogenesis of NASH: beyond steatosis and inflammation. Int J Mol Sci 2014; 15: 8591-8638.

4. Loomba R, Sanyal AJ. The global NAFLD epidemic. Nat Rev Gastroenterol Hepatol 2013; 10: 686-690.

5. Baranova A, Tran TP, Birerdinc A, Younossi ZM. Systematic review: association of polycystic ovary syndrome with metabolic syndrome and non-alcoholic fatty liver disease. Aliment Pharmacol Ther 2011; 33: 801-814.

6. Rocha ALL, Faria LC, Guimarães TCM, et al. Non-alcoholic fatty liver disease in women with polycystic ovary syndrome: systematic review and meta-analysis. J Endocrinol Invest 2017; 40: $1279-1288$.

7. De Souza LR, Berger H, Retnakaran R, et al. Non-alcoholic fatty liver disease in early pregnancy predicts dysglycemia in mid-pregnancy: prospective study. Am J Gastroenterol 2016; 111: 665-670.

8. Hagström H, Höijer J, Ludvigsson JF, et al. Adverse outcomes of pregnancy in women with non-alcoholic fatty liver disease. Liver Int 2016; 36: 268-274.

9. Sarkar M, Grab J, Dodge JL, et al. Non-alcoholic fatty liver disease in pregnancy is associated with adverse maternal and perinatal outcomes. J Hepatol 2020; 73: 516-522.

10. Castro LC, Avina RL. Maternal obesity and pregnancy outcomes. Curr Opin Obstet Gynecol 2002; 14: 601-606.

11. Page LM, Girling JC. A novel cause for abnormal liver function tests in pregnancy and the puerperium: non-alcoholic fatty liver disease. BJOG 2011; 118: 1532-1535.
12. Després JP. Abdominal obesity as important component of insulin-resistance syndrome. Nutrition 1993; 9: 452-459.

13. Meng K, Lee CH, Saremi F. Metabolic syndrome and ectopic fat deposition: what can CT and MR provide? Acad Radiol 2010; 17: 1302-1312.

14. Lee SM, Kwak SH, Koo JN, et al. Non-alcoholic fatty liver disease in the first trimester and subsequent development of gestational diabetes mellitus. Diabetologia 2019; 62: 238-248.

15. Saccone G, Khalifeh A, Al-Kouatly HB, et al. Screening for gestational diabetes mellitus: one step versus two step approach. A meta-analysis of randomized trials. J Matern Fetal Neonatal Med 2020; 33: 1616-1624.

16. Herrera JA, Arevalo-Herrera M, Herrera S. Prevention of preeclampsia by linoleic acid and calcium supplementation: a randomized controlled trial. Obstet Gynecol 1998; 91: 585-590.

17. Lopez-Jaramillo P, Barajas J, Rueda-Quijano SM, et al. Obesity and preeclampsia: common pathophysiological mechanisms. Front Physiol 2018; 9: 1838

18. Yki-Järvinen $\mathrm{H}$. Non-alcoholic fatty liver disease as a cause and a consequence of metabolic syndrome. Lancet Diabetes Endocrinol 2014; 2: 901-910.

19. Cnattingius S, Villamor E, Johansson S, et al. Maternal obesity and risk of preterm delivery. JAMA 2013; 309: 2362-2370.

20. Metzger BE, Lowe LP, Dyer AR, et al. Hyperglycemia and adverse pregnancy outcomes. N Engl J Med 2008; 358: 1991-2002.

21. Crume TL, Ogden L, West NA, et al. Association of exposure to diabetes in utero with adiposity and fat distribution in a multiethnic population of youth: the Exploring Perinatal Outcomes among Children (EPOCH) Study. Diabetologia 2011; 54: 87-92.

22. Vääräsmäki M, Pouta A, Elliot $P$, et al. Adolescent manifestations of metabolic syndrome among children born to women with gestational diabetes in a general-population birth cohort. Am J Epidemiol 2009; 169: 1209-1215.

23. Lee SM, Kim BJ, Koo JN, et al. Nonalcoholic fatty liver disease is a risk factor for large-for-gestational-age birthweight. PLoS One 2019; 14: e0221400.

24. Bril F, Ortiz-Lopez C, Lomonaco R, et al. Clinical value of liver ultrasound for the diagnosis of nonalcoholic fatty liver disease in overweight and obese patients. Liver Int 2015; 35: 2139-2146.

25. Stenberg Ribeiro M, Hagström H, Stål P, Ajne G. Transient liver elastography in normal pregnancy - a longitudinal cohort study. Scand J Gastroenterol 2019; 54: 761-765.

26. Deng D, George J, Pasupathy D, Wah Cheung N. The prevalence of metabolic associated fatty liver detected by FibroScan ${ }^{\circ}$ in women with gestational diabetes in a multiethnic population. Diabetes Res Clin Pract 2021; 174: 108757.

27. Ammon FJ, Kohlhaas A, Elshaarawy O, et al. Liver stiffness reversibly increases during pregnancy and independently predicts preeclampsia. World J Gastroenterol 2018; 24: 4393-4402.

28. Lee SS, Park SH. Radiologic evaluation of nonalcoholic fatty liver disease. World J Gastroenterol 2014; 20: 7392-7402.

29. Wong VW, Vergniol J, Wong GL, et al. Diagnosis of fibrosis and cirrhosis using liver stiffness measurement in nonalcoholic fatty liver disease. Hepatology 2010; 51: 454-462.

30. Jung YM, Lee SM, Hong S, et al. The risk of pregnancy-associated hypertension in women with nonalcoholic fatty liver disease. Liver Int 2020; 40: 2417-2426. 